\title{
Liver and spleen transient elastography predicts portal hypertension in patients with chronic liver disease: a prospective cohort study
}

\author{
Romanas Zykus ${ }^{1,2^{*}}$, Laimas Jonaitis ${ }^{1}$, Vitalija Petrenkiené ${ }^{1}$, Andrius Pranculis ${ }^{3}$ and Limas Kupčinskas ${ }^{1,2}$
}

\begin{abstract}
Background: To assess correlation between liver or spleen stiffness measurement by transient elastography (TE) and hepatic venous pressure gradient (HVPG) in patients with chronic liver disease as well find optimal and rule in/rule out cut-offs for prognosis of clinically significant (CSPH) and severe (SPH) portal hypertension.

Methods: In this prospective study patients with different chronic liver diseases were included. TE was performed at the same day prior to HVPG measurement. HVPG was measured using catheter tip occlusion technique. Based on HVPG, patients were categorized into groups of CSPH and SPH. Cut-off values were established by applying ROC curve analysis.

Results: The study included 107 consecutive patients referred for HVPG measurement or transjugular liver biopsy. Successful spleen TE was performed in 99 of the patients. Liver and spleen TE strongly correlated with HVPG, $r=0.75$ and $r=0.62$, respectively. Accuracy to detect CSPH was $88.7 \%$ for liver stiffness of $17.4 \mathrm{kPa}$ and $77.7 \%$ for spleen stiffness of $47.6 \mathrm{kPa}$. Accuracy to detect SPH was $83.1 \%$ for liver stiffness of $20.6 \mathrm{kPa}$ and $77.7 \%$ for spleen stiffness of $50.7 \mathrm{kPa}$. Liver stiffness $<11.4 \mathrm{kPa}$ could rule out CSPH with $55.2 \%$ specificity and $>21.9 \mathrm{kPa}$ rule in CSPH with $74.4 \%$ sensitivity. Liver stiffness $<12.1 \mathrm{kPa}$ could rule out SPH with $50.0 \%$ specificity and $>35 \mathrm{kPa}$ rule in SPH with $58.2 \%$ sensitivity.
\end{abstract}

Conclusions: Liver and spleen stiffness correlate with HVPG and could be used to predict CSPH or SPH. Spleen elastography was not superior to liver elastography in predicting portal hypertension.

Keywords: Transient elastography, Portal hypertension, HVPG, Fibroscan, Non-invasive test, Spleen stiffness, Liver stiffness

\section{Background}

Portal hypertension is a common and important finding in patients with progressive liver disease of any etiology, leading to further complications and decreased patient survival $[1,2]$. Direct measurement of portal hypertension is invasive and rarely used in clinical practice due to potential complications. Measurement of hepatic venous pressure gradient (HVPG) is established as a primary standard for

\footnotetext{
* Correspondence: rzykus@gmail.com

'Department of Gastroenterology, Lithuanian University of Health Sciences,

Eiveniu g. 2, Kaunas, Lithuania

${ }^{2}$ Institute for Digestive Research, Lithuanian University of Health Sciences,

Eiveniu g. 2, Kaunas, Lithuania

Full list of author information is available at the end of the article
}

evaluation of portal vein pressure, particularly in patients with hepatic (sinusoidal) portal hypertension. HVPG of less than $5 \mathrm{mmHg}$ is considered to be normal, while $6-9 \mathrm{mmHg}$ has been determined as subclinical portal hypertension [2]. It has shown that portal pressure can predict different outcomes in patients with portal hypertension. HVPG $\geq 10 \mathrm{mmHg}$ was considered as clinically significant portal hypertension (CSPH) and was linked to the risk of esophageal varices formation [3], clinical decompensation [4], development of hepatocellular carcinoma (HCC) [5] or death after liver resection due to HCC [6]. Hepatic venous pressure gradient $\geq 12 \mathrm{mmHg}$ reflected severe portal hypertension (SPH) and was found to be prognostic for acute 
variceal bleeding [7]. HVPG $\geq 16 \mathrm{mmHg}$ was the predictor of poor survival in cirrhotic patients [7]. Despite the overall safety of HVPG measurement, there are some limitations related to availability of this diagnostic procedure, personal training, experience, increased health care costs and patient discomfort. Therefore, different non-invasive tests are under investigation that potentially could replace HVPG measurement. Liver stiffness could predict portal pressure by measuring physical properties of structural changes emerging in last stages of liver fibrosis; therefore, liver elastography could be potentially accurate tool for stiffness assessment. Spleen elastography has been investigated as a tool to reflect dynamic (vascular) component next to structural part of portal hypertension. Several studies have investigated diagnostic value of liver elastography; however, the role of spleen elastography has yet to be established [8-11]. The aim of our study was to assess correlation of liver and spleen stiffness measured by transient elastography (TE) with HVPG in patients with chronic liver disease. We also aimed to find optimal liver and spleen TE values as well as rule in/rule out values in predicting $\mathrm{CSPH}$ and $\mathrm{SPH}$.

\section{Methods}

\section{Study design}

We performed a single center prospective study in the Gastroenterology Department at the Hospital of Lithuanian University of Health Sciences (Kaunas, Lithuania). Patients with different chronic liver diseases, who were referred for HVPG measurement or/and transjugular liver biopsy, were included in the study. As HPVG is an independent prognostic indicator in patients with chronic liver disease [3-7], patients were included irrespective of their liver biopsy status. Exclusion criteria was acute hepatitis, multiple focal liver lesions, cholestatic liver disease, biliary obstruction, failure to carry out liver transient elastography or patient's refusal to participate in this study. Spleen elastography was performed only if liver elastography was successful. The study was approved by local Bioethics Committee of Lithuanian University of Health Sciences (Protocol No. BE-2-26). All patients have signed an informed consent form before inclusion.

\section{Investigations}

The routine clinical (weight, height), hematological (complete blood count) and biochemical (international normalized ratio (INR), alanine aminotransferase (ALT), aspartate aminotransferase (AST), bilirubin) measurements were performed at the same day prior to HVPG measurement. Abdominal ultrasonography was performed to exclude multiple focal liver lesions.

\section{Non-invasive tests}

Liver stiffness using FIBROSCAN ${ }^{ø}$ (Echosens, Paris, France) device was measured on the same day before HVPG measurement. Patients were in fasting state. Procedure was performed in accordance with manufacturer's recommendations. Interquartile range/median $<30 \%$ and success rate $>60 \%$ was considered as good quality criteria for TE. We performed 10 successful measurements for each patient. Liver elastography was unsuccessful in 10 patients (5.9\%), who were excluded from study.

Assessment of spleen stiffness was performed by the same methodology used for liver elastography. The quality criterion (interquartile range/median, success rate and number of successful measurements) for spleen stiffness was the same as for liver stiffness. If typical elastography picture could not be found using FIBROSCAN device, exact point for spleen stiffness measurement was found using Toshiba Xario 200 ultrasound device (Toshiba Medical Systems Corporation, Japan).

\section{Hepatic venous pressure gradient}

HVPG was measured in fasting state. None of the patients have received medications affecting portal pressure before HVPG measurement. The standard criteria for HVPG measurement was applied [12]. HPVG was measured using catheter wedge technique by experienced radiologist using judkins right 6 fr catheter (Boston Scientific, USA, Marlborough). Right hepatic vein was selectively cannulated and catheter position confirmed by vein angiogram. The occluded position of the catheter was checked by absence of reflux after the injection of $2 \mathrm{~mL}$ of a contrast medium and appearance of sinusoidogram (Infinity R50, Drager, Germany). The mean of at least 3 readings was taken for further analysis. If the difference between the readings was greater than $1 \mathrm{mmHg}$, all the previous recordings were cancelled, and new readings were taken. Radiologist was blinded to clinical data and liver/spleen stiffness results.

\section{Statistical analysis}

Statistical analysis was performed using SPSS 20.0. Kolmogorov - Smirnov test was used to check data normality. For descriptive statistics frequencies, means, medians and standard deviations were calculated. HVPG scores were compared with liver and spleen stiffness expressed in $\mathrm{kPa}$ using non-parametric Spearman correlation. Based on HVPG, patients were categorized into groups with and without CSPH or into those with and without SPH. Comparisons between patients with and without CSPH or SPH were made using Mann-Whitney Test. Areas under the receiver operating characteristic (AUROC) curve were calculated and points for best specificity and sensitivity established, positive predictive value (PPV), negative predictive value (NPV) and accuracy were calculated. P-values less than 0.05 were considered to be statistically significant. 


\section{Results}

The demographic and clinical characteristics of the patients are represented in Table 1. Patients mean age was 52.3 years, with similar gender distribution. $\mathrm{HCV}$ related chronic liver disease was predominant and most patients had compensated liver disease (Child Pugh grade A).

Data of HVPG, spleen and liver elastography are represented in Table 2. Spearman correlation analysis revealed that both liver and spleen elastography correlated with hepatic venous pressure gradient. Strong correlation with HVPG was found for liver TE $(\mathrm{r}-0.75, p<0.001)$ and for spleen TE $(r-0.62, p<0.001)$. Comparisons between patients with and without CSPH or SPH was made using Mann-Whitney Test. The test revealed that there are statistically significant differences in liver and spleen stiffness between patients with and without CSPH or with and without SPH (Figs. 1 and 2).

The AUROC curves for each HVPG category are presented in Figs. 3 and 4. AUROC curve for HVPG $\geq$ $10 \mathrm{mmHg}$ was $0.949(p<0.001)$ for liver TE and 0.846 $(p<0.001)$ for spleen TE. AUROC curve for HVPG $\geq$ $12 \mathrm{mmHg}$ was $0.915(p<0.001)$ for liver TE and 0.869

Table 1 Demographic and clinical characteristics of patients

\begin{tabular}{|c|c|}
\hline & Patients $(n=107)$ \\
\hline \multicolumn{2}{|l|}{ Gender, n (\%) } \\
\hline Females & $50(46.7)$ \\
\hline Males & $57(53.3)$ \\
\hline Age, years, mean $( \pm S D)$ & $52.3( \pm 11.9)$ \\
\hline $\mathrm{BMI}, \mathrm{kg} / \mathrm{m}^{2}$, mean $( \pm \mathrm{SD})$ & $26.7( \pm 4.2)$ \\
\hline \multicolumn{2}{|l|}{ Liver disease, n (\%) } \\
\hline Hepatitis C & $68(63.6)$ \\
\hline Alcoholic liver disease & $19(17.8)$ \\
\hline Cryptogenic liver disease & $11(10.3)$ \\
\hline Other & $9(8.3)$ \\
\hline Successful spleen elastography, n (\%) & $99(92.5)$ \\
\hline \multicolumn{2}{|l|}{ HVPG, n (\%) } \\
\hline$\geq 10 \mathrm{mmHg}$ & $78(72.9)$ \\
\hline$<10 \mathrm{mmHg}$ & $29(27.1)$ \\
\hline$\geq 12 \mathrm{mmHg}$ & $67(62.6)$ \\
\hline$<12 \mathrm{mmHg}$ & $40(37.4)$ \\
\hline \multicolumn{2}{|l|}{ Child Pugh score, n (\%) } \\
\hline A & $69(64,5)$ \\
\hline B & $32(29,9)$ \\
\hline C & $1(0,9)$ \\
\hline Platelet count, $/\left\llcorner\times 10^{9}\right.$, mean $( \pm S D)$ & $130.2( \pm 75.5)$ \\
\hline ALT, IU/L, mean $( \pm S D)$ & $93.0( \pm 79.1)$ \\
\hline AST, IU/L, mean $( \pm S D)$ & $105.9( \pm 77.6)$ \\
\hline
\end{tabular}

$S D$ standard deviation, $B M I$ body mass index, HVPG hepatic venous pressure gradient, $A S T$ aspartate aminotransaminase, $A L T$ alanine aminotransaminase, INR international normalized ratio
Table 2 Descriptives of hepatic venous pressure gradient, liver and spleen transient elastography data

\begin{tabular}{llll}
\hline & HVPG $(\mathrm{mmHg})$ & Spleen TE $(\mathrm{kPa})$ & Liver TE $(\mathrm{kPa})$ \\
\hline $\mathrm{N}$ & 107 & 99 & 107 \\
Std. Error of Mean & 0,63 & 2,01 & 2,17 \\
Median & 14,0 & 62,7 & 25,7 \\
Std. Deviation & 6,5 & 20,0 & 22,4 \\
Range & 28 & 62,9 & 72,1 \\
Minimum & 1 & 12,1 & 2,9 \\
Maximum & 29 & 75,0 & 75,0 \\
\hline
\end{tabular}

HVPG hepatic venous pressure gradient, TE transient elastography

$(p<0.001)$ for spleen TE. AUROC curve values were higher for liver elastography than for spleen elastography and higher for CSPH than for SPH.

Liver and spleen TE cut-off values for different HVPG categories were established and specificity, sensitivity, positive predictive value, negative predictive value and accuracy were calculated (Table 3). To increase the practical utility of TE, we calculated cut-off point with $100 \%$ specificity (rule in) or $100 \%$ sensitivity (rule out) of liver and spleen stiffness for CSPH and SPH. The calculations revealed that there is no cut-off point with $100 \%$ specificity for spleen stiffness both in HVPG $\geq 10 \mathrm{mmHg}$ and HVPG $\geq 12 \mathrm{mmHg}$ groups. Only cut-offs with $100 \%$ sensitivity could be found, however, with little applicability. Spleen stiffness of $14.3 \mathrm{kPa}$ had $4.2 \%$ sensitivity for detecting HVPG $\geq 10 \mathrm{mmHg}$, while spleen stiffness of $32.6 \mathrm{kPa}$ had $48.6 \%$ sensitivity for HVPG $\geq 12 \mathrm{mmHg}$. In contrast to spleen TE data, analysis of liver stiffness revealed cut-off points with $100 \%$ specificity or sensitivity in all groups (Table 4).

\section{Discussion}

Up to date, several studies that evaluated liver elastography for prediction of clinically significant portal hypertension have been published [9-11, 13-17]. In these studies AUROC curve for prediction of CSPH varies between 0.81 and 0.94 with optimal cut-offs between 16.8 and $21.95 \mathrm{kPa}$ with sensitivity and specificity of 73.7 $-89.7 \%$ and $73.7-82.2 \%$, respectively $[9,13-15]$. Both AUROC curves and optimal cut-offs observed by other authors are comparable with our data that show AUROC of 0.949 and optimal cut-off $17.4 \mathrm{kPa}$ with $88 \%$ sensitivity and $87.5 \%$ specificity. Nevertheless, applicability of optimal cut-off points is questionable due to the risk of patient misclassification; therefore, cut-off points with $100 \%$ sensitivity or specificity could be more useful to assess portal hypertension non-invasively, especially in HCC patients referred for surgery for evaluation of contraindications to perform liver resection. It was found that liver stiffness $>29.0 \mathrm{kPa}$ predicts $\mathrm{CSPH}$ with $71.9 \%$ sensitivity and $100 \%$ specificity [10]. Similar 


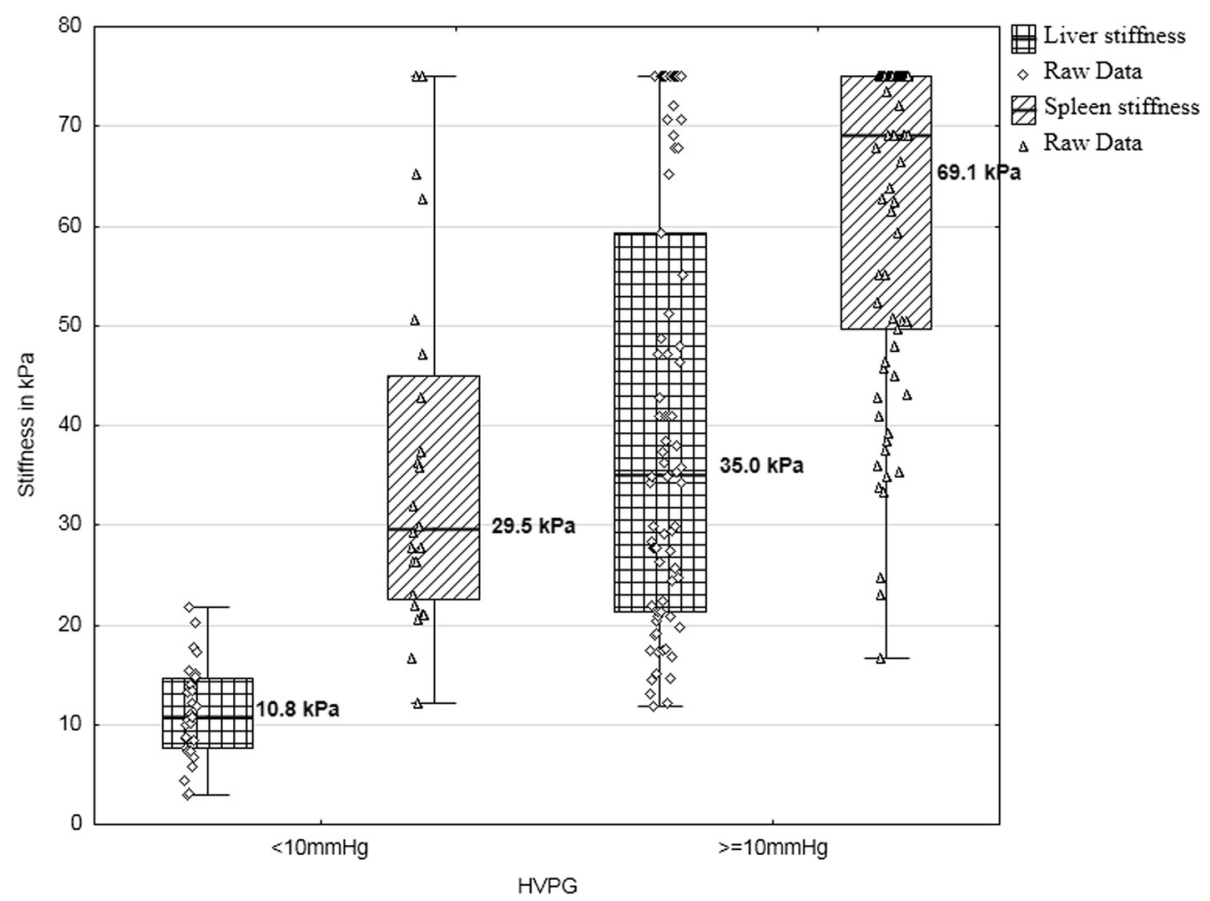

Fig. 1 The comparison of median liver and spleen stiffness in patients with and without clinically significant portal hypertension ( $\geq 10 \mathrm{mmHg}$; $p$-value $<0.01$ for differences in liver stiffness; $p$-value $<0.01$ for differences in spleen stiffness

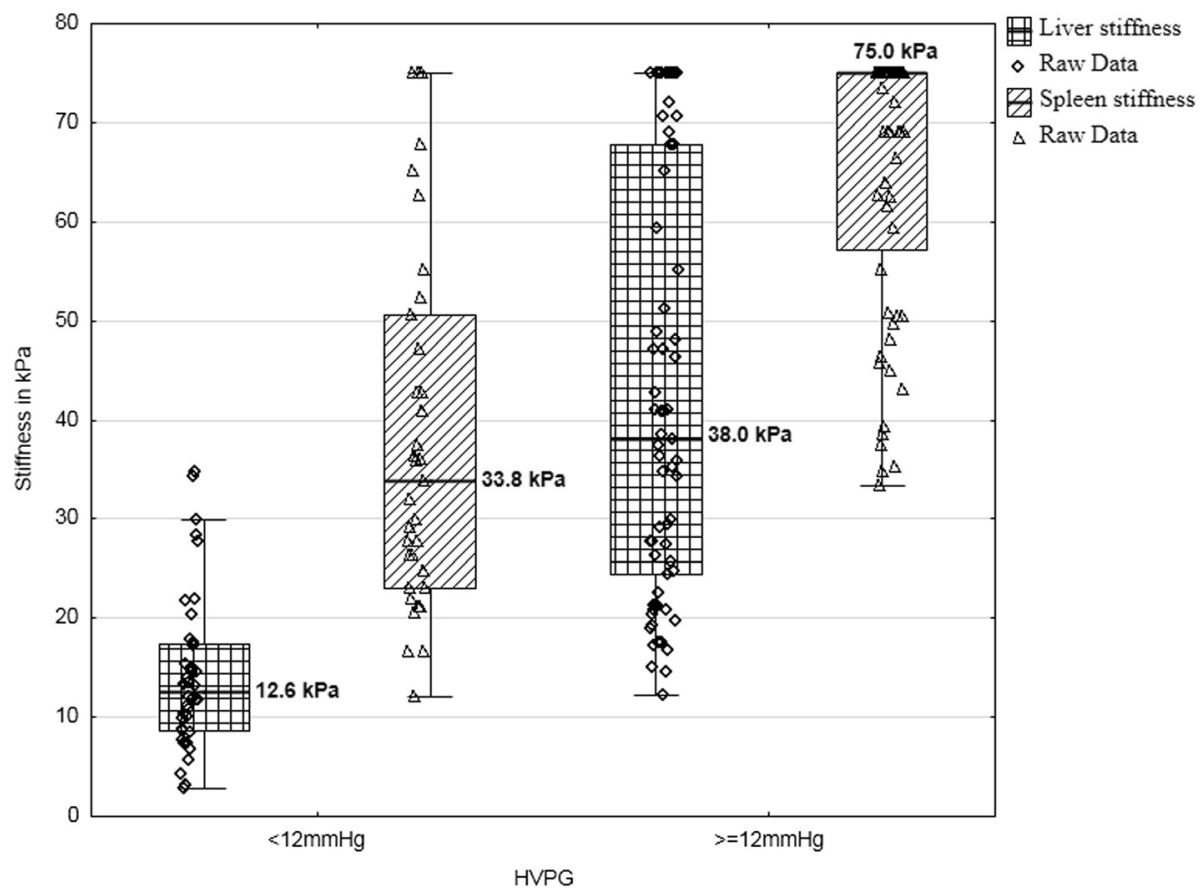

Fig. 2 Liver and spleen stiffness comparison in patients with and without severe portal hypertension $(\geq 12 \mathrm{mmHg})$; $p$-value $<0.01$ for differences in liver stiffness; $p$-value $<0.01$ for differences in spleen stiffness 


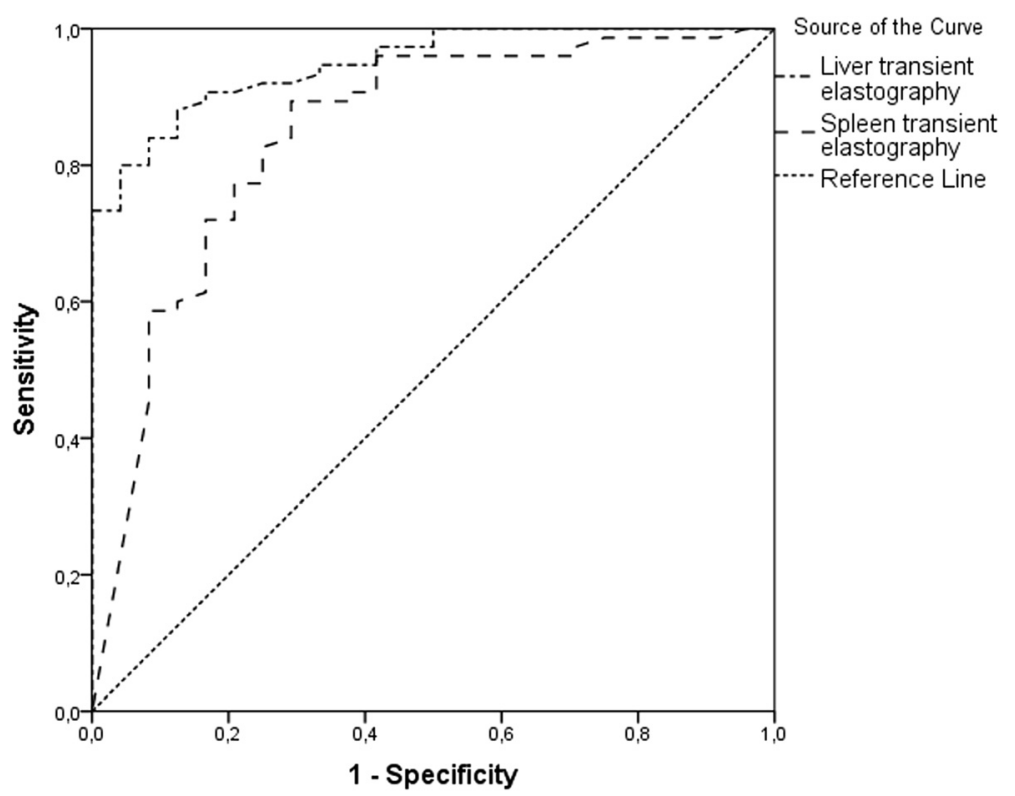

Fig. 3 AUROC curves of liver and spleen transient elastography for clinically significant portal hypertension $(\geq 10 \mathrm{mmHg})$

observations were made in other studies where liver stiffness of $>21 \mathrm{kPa}$ in $\mathrm{HCV}$ patients predicted $\mathrm{CSPH}$ with sensitivity $42 \%$ and specificity of $100 \%$ [16] or $\geq 24.2 \mathrm{kPa}$ with sensitivity $52.3 \%$ and specificity $97.1 \%$ [18]. The rule out cut-off point was found to be $<16 \mathrm{kPa}$ with sensitivity $95.4 \%$ and specificity $68.6 \%$. Our data revealed comparable cut-off of $21.9 \mathrm{kPa}$ with sensitivity of $74.4 \%$ and specificity of $100 \%$. Combining these results with the threshold of $11.4 \mathrm{kPa}$ (a measure close to liver cirrhosis stage) with sensitivity of $100 \%$ and specificity of $55.2 \%$, we can determine the "grey zone" between 11.4 and $21.9 \mathrm{kPa}$, and correctly classify patients outside of it. This strategy could be implemented into decision making for HCC patients, were HVPG $\geq$ $10 \mathrm{mmHg}$ predicts higher mortality and liver dysfunction after liver resection. This group of patients could avoid unnecessary HVPG measurement if TE measurement is outside the "grey zone".

Few studies investigated liver TE in SPH, where AUROC curve was found to be between 0.79 and 0.92

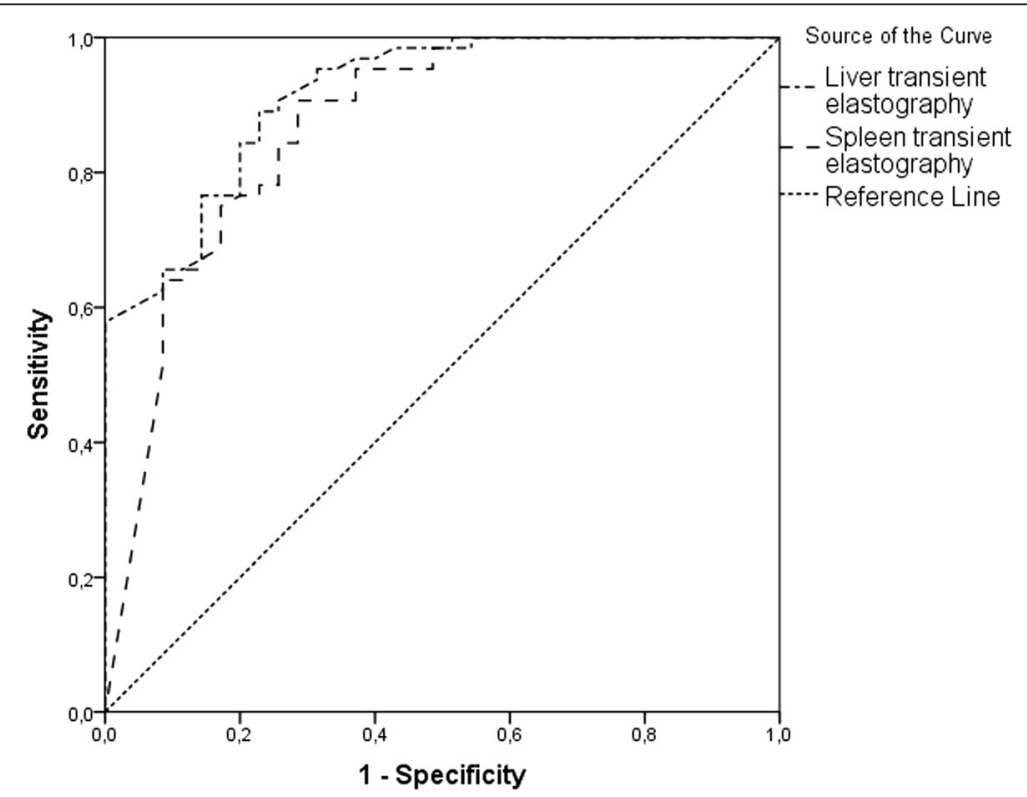

Fig. 4 AUROC curves of liver and spleen transient elastography for severe portal hypertension ( $\geq 12 \mathrm{mmHg}$ ) 
Table 3 Optimal cut-offs of liver and spleen stiffness for prediction of clinically significant ( $\geq 10 \mathrm{mmHg})$ and severe $(\geq 12 \mathrm{mmHg})$ portal hypertension

\begin{tabular}{|c|c|c|c|c|c|c|c|}
\hline$\overline{H V P G}$ & Method & Cut-off, kPa & Sensitivity, \% & Specificity, \% & PPV, \% & NPV, \% & Accuracy, \% \\
\hline \multirow[t]{2}{*}{$\geq 10 \mathrm{mmHg}$} & Liver TE & 17.4 & 88.0 & 87.5 & 95.8 & 74.2 & 88.7 \\
\hline & Spleen TE & 47.6 & 77.3 & 79.2 & 92.0 & 52.7 & 77.7 \\
\hline \multirow[t]{2}{*}{$\geq 12 \mathrm{mmHg}$} & Liver TE & 20.6 & 82.8 & 80.0 & 88.8 & 0.75 & 83.1 \\
\hline & Spleen TE & 50.7 & 78.1 & 77.1 & 86.2 & 65.8 & 77.7 \\
\hline
\end{tabular}

HVPG hepatic venous pressure gradient, PPV positive predictive value, NPV negative predictive value

$[13,15,19,20]$. The optimal cut-offs to predict SPH varied between 17.6 and $24.2 \mathrm{kPa}$ with sensitivity and specificity of $82.9-94 \%$ and $66.6-81 \%$, respectively $[13,15,19,20]$. Our study revealed comparable results with optimal cut-off $20.6 \mathrm{kPa}$. Considering marginal cutoffs, our study revealed that rule out cut-off of $12.1 \mathrm{kPa}$ had $50 \%$ specificity, though it was almost the same as in CSPH group. Rule in cut-off point of $35.0 \mathrm{kPa}$ had sensitivity of $58.2 \%$ and it is further than CSPH cut-off.

Studies analyzing correlation of spleen stiffness measured by transient elastography with HVPG are still scarce. Colechia et al. found strong correlation between spleen stiffness and HVPG $(\mathrm{r}-0.88)$ [18]. The marginal cut-off points were calculated to rule in or rule out $\mathrm{CSPH}$ or SPH. For CSPH cut-off $40.0 \mathrm{kPa}$ had sensitivity of $98.5 \%$ and specificity of $74.3 \%$ and cut-off $52.8 \mathrm{kPa}$ $76.9 \%$ and $97.1 \%$, respectively. For SPH cut-off 41.3 $\mathrm{kPa}$ had sensitivity of $98.1 \%$ and specificity of $67.4 \%$ and cut-off $55.0 \mathrm{kPa}-72.2 \%$ and $97.8 \%$, respectively. Our study did not show clinically useful rule in and rule out points obtained by measurement of spleen stiffness, because of wide scatter and overlap of spleen elastography in $\mathrm{CSPH}$ and $\mathrm{SPH}$ groups. In comparison, the cutoff $40 \mathrm{kPa}$ in our cohort had sensitivity of $85 \%$ and specificity of $70 \%$ and cut-off $53.0 \mathrm{kPa} 69.3 \%$ and $83.3 \%$, respectively, for CSPH. Another study found no significant differences in spleen stiffness measured by TE between groups analyzing $\mathrm{CSPH}$ or $\mathrm{SPH}$ [8], although the study was underpowered including only 35 patients with just three patients without CSPH.

Our data show that liver and spleen elastography correlate well with HVPG, however liver stiffness has better correlation coefficient and AUROC than spleen

Table 4 Rule in and rule out cut-offs of liver stiffness for prediction of clinically significant $(\geq 10 \mathrm{mmHg}$ ) and severe $(\geq 12 \mathrm{mmHg}$ ) portal hypertension

\begin{tabular}{lllll}
\hline HVPG & Method & Cut-off, kPa & Sensitivity, \% & Specificity, \% \\
\hline$\geq 10 \mathrm{mmHg}$ & Liver TE & 11.4 & 100 & 55.2 \\
& & 21.9 & 74.4 & 100 \\
$212 \mathrm{mmHg}$ & \multirow{2}{*}{ Liver TE } & 12.1 & 100 & 50.0 \\
& & 35.0 & 58.2 & 100 \\
\hline
\end{tabular}

HVPG hepatic venous pressure gradient, TE transient elastography elastography. We expected that spleen elastography might be more accurate than liver elastography for evaluation of HVPG subgroups because it reflects dynamic component of portal hypertension. Insufficient accuracy of spleen TE could be partially explained by influence of various shunts, arising during progression of portal hypertension. Taking into account other drawbacks of spleen elastography such as decreased success of spleen TE performance, missing cut-off points with $100 \%$ specificity or sensitivity and less standardized procedure, it appears to suggest little benefit in clinical practice and no significant advantage over liver elastography. The wide variation of liver transaminases in our patients cohort could lead to higher transient elastography values and consequently to higher cut-off, as noted in different studies analyzing correlation of TE with liver fibrosis [21]. However, diagnostic accuracy of TE for cirrhosis stage is considered reliable and the influence of inflammation is less pronounced [21, 22]. Although liver biopsy was performed just in half of our patients, most of them had cirrhosis or pre-cirrhosis stage and all patients without biopsy where diagnosed as having cirrhosis based on laboratory, radiologic and clinical features. Therefore, we think that liver transaminases did not introduce significant bias into our data. To decrease the influence of inflammation to our data, we excluded all patients with acute and acute on chronic hepatitis. In our study most of the patients belonged to Child A class. Inclusion of more advanced stages of liver cirrhosis in such studies is often complicated by the presence of ascites which reflects one of the limitations of TE. The benefit of spleen stiffness measurement for evaluation of prognosis in patients with portal hypertension still has to be determined in further studies. The limitations of TE potentially could be resolved using other elastography types such as Acoustic Radiation Force Impulse imaging or Shear Wave Elastography. Further studies have to evaluate whether spleen elastography could be superior to liver elastography with these new elastography techniques for non-invasive estimation of portal hypertension [23].

\section{Conclusions}

Our study revealed that both liver and spleen stiffness measured by transient elastography correlate with hepatic 
venous pressure gradient. However, spleen elastography has no advantage in comparison to standard liver elastography for prediction of clinically significant or severe portal hypertension. Liver stiffness $<11.4 \mathrm{kPa}$ could rule out and $>21.9 \mathrm{kPa}$ rule in clinically significant portal hypertension. Liver stiffness $<12.1 \mathrm{kPa}$ could rule out and $>35 \mathrm{kPa}$ rule in severe portal hypertension.

\section{Abbreviations}

CSPH: Clinically significant portal hypertension; HVPG: Hepatic venous pressure gradient; SPH: Severe portal hypertension; HCC: Hepatocellular carcinoma; TE: Transient elastography; INR: International normalized ratio; ALT: Alanine aminotransferase; AST: Aspartate aminotransferase; AUROC: Area under the receiver operating characteristic; PPV: Positive predictive value; NPV: Negative predictive value; SD: Standard deviation; BMI: Body mass index.

\section{Competing interests}

The authors state no conflict of interest.

\section{Authors' contributions}

$L K, R Z, L, V P$ designed the study and analyzed the data. RZ, VP and AP included patients into study and collected data. RZ performed liver elastography. AP performed HVPG examination. RZ, L, VP, AP and LK participated in the drafting of the manuscript. $\amalg$ and $L K$ have given final approval of the version to be published. All authors read and approved the final manuscript.

\section{Acknowledgements}

The authors declare that the manuscript has not been supported by any sources of support, including sponsorship and any sources of material.

\section{Author details}

'Department of Gastroenterology, Lithuanian University of Health Sciences, Eiveniu g. 2, Kaunas, Lithuania. ${ }^{2}$ Institute for Digestive Research, Lithuanian University of Health Sciences, Eiveniu g. 2, Kaunas, Lithuania. ${ }^{3}$ Department of Radiology, Lithuanian University of Health Sciences, Eiveniu g. 2, Kaunas, Lithuania.

Received: 1 July 2015 Accepted: 18 December 2015 Published online: 24 December 2015

\section{References}

1. Ripoll C, Bañares R, Rincón D, Catalina M-V, Lo lacono O, Salcedo M, et al. Influence of hepatic venous pressure gradient on the prediction of survival of patients with cirrhosis in the MELD Era. Hepatology. 2005;42:793-801.

2. Berzigotti A, Seijo S, Reverter E, Bosch J. Assessing portal hypertension in liver diseases. Expert Rev Gastroenterol Hepatol. 2013;7:141-55.

3. Groszmann RJ, Garcia-Tsao G, Bosch J, Grace ND, Burroughs AK, Planas R, et al. Beta-blockers to prevent gastroesophageal varices in patients with cirrhosis. N Engl J Med. 2005;353:2254-61.

4. Ripoll C, Groszmann R, Garcia-Tsao G, Grace N, Burroughs A, Planas R, et al. Hepatic venous pressure gradient predicts clinical decompensation in patients with compensated cirrhosis. Gastroenterology. 2007;133:481-8.

5. Ripoll C, Groszmann RJ, Garcia-Tsao G, Bosch J, Grace N, Burroughs A, et al. Hepatic venous pressure gradient predicts development of hepatocellular carcinoma independently of severity of cirrhosis. J Hepatol. 2009;50:923-8.

6. Boleslawski E, Petrovai G, Truant S, Dharancy S, Duhamel A, Salleron J, et al. Hepatic venous pressure gradient in the assessment of portal hypertension before liver resection in patients with cirrhosis. $\mathrm{Br} J$ Surg. 2012:99:855-63.

7. Stanley AJ, Robinson I, Forrest EH, Jones AL, Hayes PC. Haemodynamic parameters predicting variceal haemorrhage and survival in alcoholic cirrhosis. QJM. 1998;91:19-25.

8. Elkrief L, Rautou P-E, Ronot M, Lambert S, Dioguardi Burgio M, Francoz C, et al. Prospective Comparison of Spleen and Liver Stiffness by Using ShearWave and Transient Elastography for Detection of Portal Hypertension in Cirrhosis. Radiology. 2014;275:289-98.
9. Bureau C, Metivier S, Peron JM, Selves J, Robic MA, Gourraud PA, et al, Transient elastography accurately predicts presence of significant portal hypertension in patients with chronic liver disease. Aliment Pharmacol Ther 2008;27:1261-8

10. Kitson MT, Roberts SK, Colman JC, Paul E, Button P, Kemp W. Liver stiffness and the prediction of clinically significant portal hypertension and portal hypertensive complications. Scand J Gastroenterol. 2015;50:462-9.

11. Lemoine M, Katsahian S, Ziol M, Nahon P, Ganne-Carrie N, Kazemi F, et al. Liver stiffness measurement as a predictive tool of clinically significant portal hypertension in patients with compensated hepatitis $C$ virus or alcohol-related cirrhosis. Aliment Pharmacol Ther. 2008;28:1102-10.

12. Groszmann RJ, Wongcharatrawee $\mathrm{S}$. The hepatic venous pressure gradient: anything worth doing should be done right. Hepatology. 2004;39:280-2.

13. Reiberger T, Ferlitsch A, Payer BA, Pinter M, Schwabl P, Stift J, et al. Noninvasive screening for liver fibrosis and portal hypertension by transient elastography-a large single center experience. Wien Klin Wochenschr. 2012;124:395-402

14. Salzl $P$, Reiberger $T$, Ferlitsch $M$, Payer BA, Schwengerer $B$, Trauner $M$, et al Evaluation of portal hypertension and varices by acoustic radiation force impulse imaging of the liver compared to transient elastography and AST to platelet ratio index. Ultraschall Med. 2014;35:528-33.

15. Hong WK, Kim MY, Baik SK, Shin SY, Kim JM, Kang YS, et al. The usefulness of non-invasive liver stiffness measurements in predicting clinically significant portal hypertension in cirrhotic patients: Korean data. Clin Mol Hepatol. 2013;19:370-5.

16. Llop E, Berzigotti A, Reig M, Erice E, Reverter E, Seijo S, et al. Assessment of portal hypertension by transient elastography in patients with compensated cirrhosis and potentially resectable liver tumors. J Hepatol. 2012;56:103-8.

17. Carrión JA, Navasa M, Bosch J, Bruguera M, Gilabert R, Forns X. Transient elastography for diagnosis of advanced fibrosis and portal hypertension in patients with hepatitis $C$ recurrence after liver transplantation. Liver Transpl. 2006;12:1791-8.

18. Colecchia A, Montrone L, Scaioli E, Bacchi-Reggiani ML, Colli A, Casazza G, et al. Measurement of spleen stiffness to evaluate portal hypertension and the presence of esophageal varices in patients with HCV-related cirrhosis. Gastroenterology. 2012;143:646-54.

19. Sánchez-Conde M, Miralles P, Bellón JM, Rincón D, Ramírez M, Gutiérrez I, et al. Use of transient elastography (FibroScan ${ }^{\oplus}$ ) for the noninvasive assessment of portal hypertension in HIV/HCV-coinfected patients. J Viral Hepat. 2011;18:685-91.

20. Vizzutti F, Arena U, Romanelli RG, Rega L, Foschi M, Colagrande S, et al. Liver stiffness measurement predicts severe portal hypertension in patients with HCV-related cirrhosis. Hepatology. 2007:45:1290-7.

21. Tsochatzis EA, Gurusamy KS, Ntaoula S, Cholongitas E, Davidson BR, Burroughs AK. Elastography for the diagnosis of severity of fibrosis in chronic liver disease: a meta-analysis of diagnostic accuracy. J Hepatol. 2011;54:650-9.

22. Tapper EB, Cohen EB, Patel K, Bacon B, Gordon S, Lawitz E, et al. Levels of Alanine Aminotransferase Confound Use of Transient Elastography to Diagnose Fibrosis in Patients With Chronic Hepatitis C Virus Infection. Clin Gastroenterol Hepatol. 2012;10:932-7. e1.

23. Rifai K, Cornberg J, Bahr M, Mederacke I, Potthoff A, Wedemeyer H, et al. ARFI elastography of the spleen is inferior to liver elastography for the detection of portal hypertension. Ultraschall Med. 2011;32 Suppl 2:E24-30.

Submit your next manuscript to BioMed Central and we will help you at every step:

- We accept pre-submission inquiries

- Our selector tool helps you to find the most relevant journal

- We provide round the clock customer support

- Convenient online submission

- Thorough peer review

- Inclusion in PubMed and all major indexing services

- Maximum visibility for your research

Submit your manuscript at www.biomedcentral.com/submit 